\title{
Mictomagnetic Order in $\mathrm{Cd}_{0.87} \mathrm{Cr}_{1.93} \mathrm{~V}_{0.06} \mathrm{Se}_{4}$ Semiconductor
}

\author{
T. Groń ${ }^{a}$, E. MalickA ${ }^{b}$, B. ZaWisza ${ }^{b}$, H. Duda ${ }^{a}$, J. Krok-Kowalski $^{a}$ \\ AND A.W. PACYNA ${ }^{c}$ \\ ${ }^{a}$ University of Silesia, Institute of Physics, Uniwersytecka 4, 40-007 Katowice, Poland \\ ${ }^{b}$ University of Silesia, Institute of Chemistry, Szkolna 9, 40-006 Katowice, Poland \\ ${ }^{c}$ The Henryk Niewodniczański Institute of Nuclear Physics, Polish Academy of Sciences \\ E. Radzikowskiego 152, 31-342 Kraków, Poland
}

\begin{abstract}
The complex ac dynamic magnetic susceptibility was used to study the mictomagnetic-like behavior in polycrystalline $\mathrm{Cd}_{0.87} \mathrm{Cr}_{1.93} \mathrm{~V}_{0.06} \mathrm{Se}_{4}$ spinel. The temperature dependences of the zero field in-phase (real part) and out-of-phase (imaginary part) components of fundamental susceptibility measured at the oscillating field $H_{\mathrm{ac}}=0.5 \mathrm{Oe}$ and at the constant frequency of $125 \mathrm{~Hz}$ are characteristic for the mictomagnetic order. These results well correlate with the anomalies in the second and third harmonics of the ac susceptibility indicating the cluster glass.
\end{abstract}

PACS: 72.80.Jc, 75.50.Pp, 75.50.Dd

\section{Introduction}

The electrical and magnetic investigations carried out on the polycrystalline spinels with the general chemical formula $\mathrm{Cd}_{x} \mathrm{Cr}_{y} \mathrm{~V}_{z} \mathrm{Se}_{4}$ (where $z=0.06,0.12,0.24$, and 0.31 ) revealed semiconducting and ferromagnetic properties with an almost constant Curie temperature $T_{\mathrm{C}}=128 \mathrm{~K}$ and a Curie-Weiss temperature $\left(\theta_{\mathrm{CW}}\right)$ decreasing from $160 \mathrm{~K}$ for $z=0.06$ to $140 \mathrm{~K}$ for $z=0.31$ [1]. The $\mathrm{Cd}_{0.87} \mathrm{Cr}_{1.93} \mathrm{~V}_{0.06} \mathrm{Se}_{4}$ spinel pays special attention because it showed a change of thermopower sign from $p$ to $n$ at $243 \mathrm{~K}$ and a step-like structure of the electrical conductivity (SLS EC). This structure has been explained in a framework of the impurity conduction and hopping conduction as well as with the formation of ferromagnetic clusters in the $\mathrm{Cr}$ band, which is partly imperfect by cation deficiency from one side and weakly diluted by the $\mathrm{V}^{3+}$ ions forming the $3 d^{2} t_{2 \mathrm{~g}}^{2} e_{\mathrm{g}}^{0}$ band from the other [1]. The SLS EC effect was first discovered in the $\mathrm{Zn}_{1-x} \mathrm{Ga}_{2 x / 3} \mathrm{Cr}_{2} \mathrm{Se}_{4}$ spinel system for the single crystal containing $3 \% \mathrm{Ga}$ ions [2] and next in the $\mathrm{CdCr}_{2-x} \mathrm{Ga}_{x} \mathrm{Se}_{4}$ spinel system for the single crystal containing $1.5 \%$ Ga ions occupied in both cases the octahedral sites [3]. Pure $\mathrm{CdCr}_{2} \mathrm{Se}_{4}$ combines a $p$-type semiconducting behaviour of the Arrhenius type and ferromagnetic order via superexchange interaction with $T_{\mathrm{C}}=142 \mathrm{~K}$ and $\theta_{\mathrm{CW}}=190 \mathrm{~K}$ [4].

The main purpose of the present work is an attempt to study in more detail a magnetic order and correlate it with the SLS EC phenomenon of the polycrystalline $\mathrm{Cd}_{0.87} \mathrm{Cr}_{1.93} \mathrm{~V}_{0.06} \mathrm{Se}_{4}$.

\section{Experimental details}

Powder sample of the $\mathrm{Cd}_{0.87} \mathrm{Cr}_{1.93} \mathrm{~V}_{0.06} \mathrm{Se}_{4}$ spinel was obtained by a ceramic method [5]. The X-ray diffraction and a precise atomic content determination were done with the aid of the SIEMENS D5000 diffractometer and the energy-dispersive X-ray spectrometry (EDXRF), respectively, showing a single-phase material with a cubic spinel structure $(F d 3 m)$ and a lattice parameter of $1074.70 \mathrm{pm}$. The composition studied in this paper is the same as reported in Ref. [1].

The dynamic ac magnetic susceptibilities using a Lake Shore 7225 ac susceptometer were measured in the zero-field-cooling mode. The in-phase $\chi_{1}^{\prime}$ and out-of-phase $\chi_{1}^{\prime \prime}$ components of the ac fundamental susceptibility were recorded in the temperature range $4.2-150 \mathrm{~K}$ simultaneously as a function of temperature in an oscillating field $H_{\mathrm{ac}}=0.5$ Oe with frequency of $125 \mathrm{~Hz}$ and in the different external magnetic fields $H_{\mathrm{dc}}$ changing from 0 to $5 \mathrm{kOe}$. The signals of the second $\left(\chi_{2}\right)$ and third $\left(\chi_{3}\right)$ harmonics associated with nonlinear susceptibilities were detected as a function of temperature using an oscillating field of 1 Oe without the applied external magnetic field.

\section{Results and discussion}

Figure 1 shows the temperature dependence of the dynamic magnetic susceptibility recorded at $H_{\mathrm{ac}}=0.5 \mathrm{Oe}$ with frequency $f=125 \mathrm{~Hz}$. For $H_{\mathrm{dc}}=0$ the $\chi_{1}^{\prime}(T)$ shows a linear increase up to $50 \mathrm{~K}$, the paraprocess in the range $50-100 \mathrm{~K}$, a constant value in the range 100-125, and a sharp drop at $T_{\mathrm{C}}=128 \mathrm{~K}$. With increasing $H_{\mathrm{dc}}$ from 100 to $5 \mathrm{kOe}$ the $\chi_{1}^{\prime}$ intensity suppresses, $T_{\mathrm{C}}$ shifts to higher temperatures and simultaneously a broad hump occurs with a maximum shifting to lower temperatures. The $\chi_{1}^{\prime \prime}(T)$ reveals the largest energy lost in the range $4.2-50 \mathrm{~K}$ with maximum at $20 \mathrm{~K}$ slightly shifting to lower temperatures as $H_{\mathrm{dc}}$ increases. The $\chi_{1}^{\prime}(T)$ dependence seems to be attributed to the cluster glass-like behaviour 


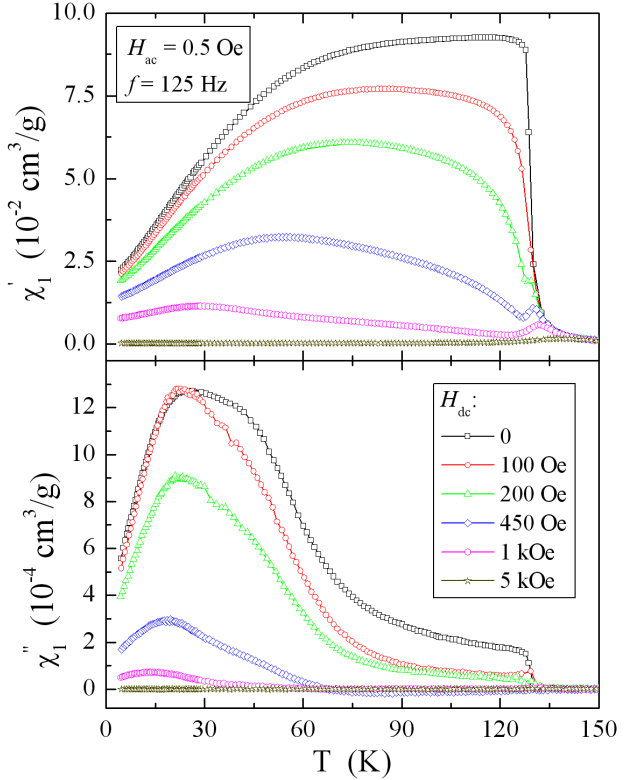

Fig. 1. In-phase $\chi_{1}^{\prime}$ and out-of-phase $\chi_{1}^{\prime \prime}$ components of zero field fundamental susceptibility vs. temperature $T$ recorded at $H_{\mathrm{ac}}=0.5$ Oe with $f=125 \mathrm{~Hz}$ for external magnetic fields $H_{\mathrm{dc}}$ changing from 0 to $5 \mathrm{kOe}$.

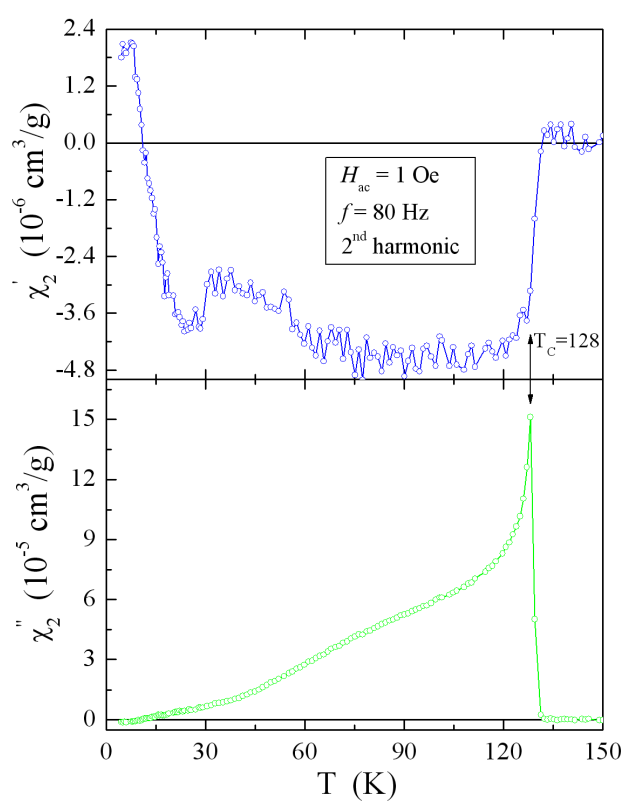

Fig. 2. In-phase $\chi_{2}^{\prime}$ and out-of-phase $\chi_{2}^{\prime \prime}$ components of second harmonics of zero field susceptibility vs. temperature $T$ recorded at $H_{\mathrm{ac}}=1$ Oe with $f=80 \mathrm{~Hz}$.

where the local correlations of the magnetic ions (as pairs, triplets, and so forth) are coupled by direct exchange [6].

Figures 2 and 3 show the higher harmonics of dynamic susceptibility, i.e. the second $\chi_{2}(T)$ and third $\chi_{3}(T)$ which have one feature in common: they vanish above $T_{\mathrm{C}}$ in accordance with the simple molecular field the-

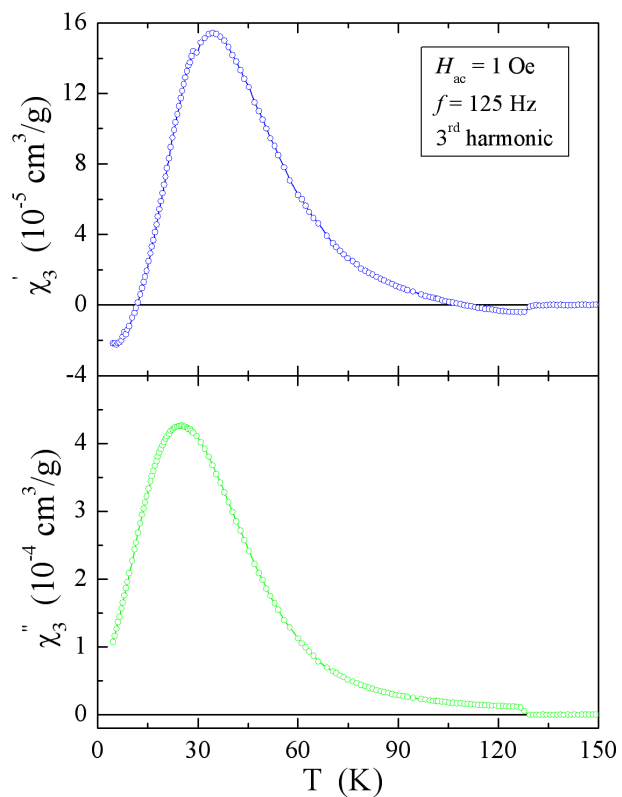

Fig. 3. In-phase $\chi_{3}^{\prime}$ and out-of-phase $\chi_{3}^{\prime \prime}$ components of third harmonics of zero field susceptibility vs. temperature $T$ recorded at $H_{\mathrm{ac}}=1$ Oe with $f=125 \mathrm{~Hz}$.

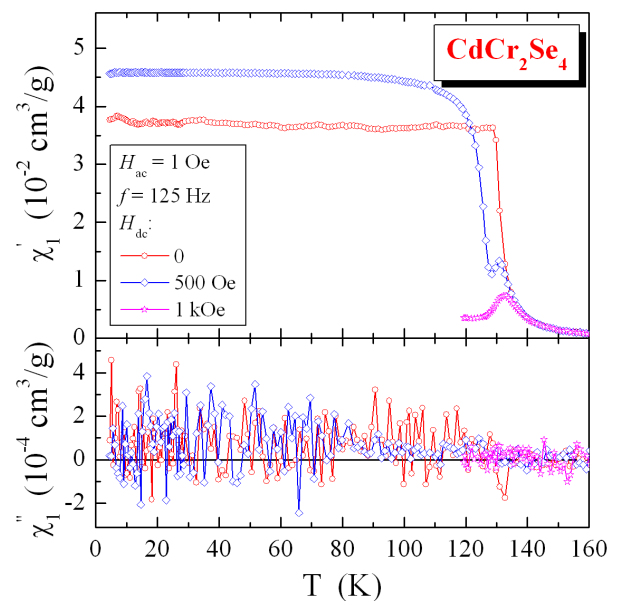

Fig. 4. In-phase $\chi_{1}^{\prime}$ and out-of-phase $\chi_{1}^{\prime \prime}$ components of zero field fundamental susceptibility vs. temperature $T$ for $\mathrm{CdCr}_{2} \mathrm{Se}_{4}$ recorded at $H_{\mathrm{ac}}=1$ Oe with $f=125 \mathrm{~Hz}$ for external magnetic fields $H_{\mathrm{dc}}$ changing from 0 to $1 \mathrm{kOe}$.

ory [7]. The in-phase second $\chi_{2}^{\prime}$ and third $\chi_{3}^{\prime}$ harmonics change their signs in opposite way both at $15 \mathrm{~K}$ and $T_{\mathrm{C}}$. Next, the out-of-phase second $\chi_{2}^{\prime \prime}$ and third $\chi_{3}^{\prime \prime}$ harmonics reveal the energy dissipation at $T_{\mathrm{C}}$ and $15 \mathrm{~K}$, respectively. The negative value of the real part and the positive value of the imaginary part of the 2nd harmonic of the ac magnetic susceptibility (Fig. 2) are the experimental signatures of the spin glass state. Here, the partially destroyed ferromagnetic (via superexchange) interaction may result purely from the geometry or topology of the lattice (known as the geometric frustration). This is con- 
nected mainly with the $\mathrm{V}$ substitution, because the $\mathrm{V}$ ion has a larger radius $(64 \mathrm{pm})$ and smaller magnetic moment in comparison with the $\mathrm{Cr}$ one (61.5 pm [8]).

Figure 4 shows the temperature dependence of the dynamic magnetic susceptibility of the $\mathrm{CdCr}_{2} \mathrm{Se}_{4}$ matrix recorded at $H_{\mathrm{ac}}=1$ Oe with frequency $f=125 \mathrm{~Hz}$ for comparison. For $H_{\mathrm{dc}}=0$ the $\chi_{1}^{\prime}$ reveals typical ferromagnetic order and the $\chi_{1}^{\prime \prime}$ oscillates close to zero indicating a lack of the energy dissipation. A striking difference between the $\chi_{1}^{\prime}(T)$ dependences in Figs. 1 and 4 is evident. On the other hand, $\chi_{1}^{\prime}$ shows a peak near the Curie temperature when heating the sample in a fixed (small) magnetic field [9]. This is characteristic for the Hopkinson effect in the samples consisting of fine powders of single domain particles [10, 11].

\section{Conclusions}

It is clearer now that the mictomagnetic order in the $\mathrm{Cd}_{0.87} \mathrm{Cr}_{1.93} \mathrm{~V}_{0.06} \mathrm{Se}_{4}$ can occur and the small groups around the $\mathrm{V}$ ions coexist as the spin-glass entities embedded in a ferromagnetic matrix. From the other hand, Monte Carlo simulations carried out on large samples of two-dimensional and three-dimensional Ising models confirmed that for critical concentrations (i.e., for the fraction of negative interactions and for the fraction of frustrated spins) the magnetic defect structures (clusters) appear leading to the unusual changes of the physical properties [12]. Thus, a mictomagnetic order, i.e. a cluster glass maybe the main reason of the appearance of a step-like structure of the electrical conductivity experimentally observed in the studied spinel.

One can conclude that a presence of a few percent of the $\mathrm{V}$ ions in the octahedral positions makes easier a rapid increase in conductivity with increasing tempera- ture as well as a dissolution of the ferromagnetic spin clusters on going towards the paramagnetic limit.

\section{Acknowledgments}

This work is partly founded from science grant No. N N204 145938.

\section{References}

[1] T. Groń, H. Duda, E. Malicka, B. Zawisza, J. Krok-Kowalski, A.W. Pacyna, Acta Phys. Pol. A 116, 969 (2009).

[2] T. Groń, E. Malicka, I. Okońska-Kozłowska, A. Waśkowska, in: Ferrites: Proc. Eight Int. Conf. on Ferrites, The Japan Society of Powder and Powder Metallurgy, Kyoto 2000, p. 211.

[3] T. Groń, A. Krajewski, J. Kusz, E. Malicka, I. Okońska-Kozłowska, A. Waśkowska, Phys. Rev. B 71, 035208 (2005).

[4] P.K. Baltzer, H.W. Lehmann, M. Robbins, Phys. Rev. Lett. 15, 493 (1965).

[5] I. Okońska-Kozłowska, J. Krok, Z. Anorg. Allg. Chem. 447, 235 (1978).

[6] C.M. Hurd, Contemp. Phys. 23, 469 (1982).

[7] T. Hashimoto, A. Sato, Y. Fujiwara, J. Phys. Soc. Jpn. 35, 81 (1973).

[8] R.D. Shannon, Acta Crystallogr. A 32, 751 (1976).

[9] J. Hopkinson, Proc. R. Soc. Lond. 48, 1 (1890).

[10] O. Popov, M. Mikhov, J. Magn. Magn. Mater. 75 , 135 (1988).

[11] O. Popov, P. Rachev, M. Mikhov, F. Calderon, J.L. Sanchez, F. Leccabue, J. Magn. Magn. Mater. 99, 119 (1991).

[12] S. Kirkpatrick, Phys. Rev. B 16, 4630 (1977). 